\title{
Selective Small Reconstruction Error Based LDRC Multimodal Biometric Authentication
}

\author{
Savitha G. ${ }^{*}$, Vibha L. $^{2}$, Venugopal K. R. ${ }^{3}$ \\ ${ }^{1 *}$ Dept. Computer Science and Engineering, B.N.M. Institute of Technology, Bangalore, India \\ ${ }^{2}$ Dept. Computer Science and Engineering, B.N.M. Institute of Technology, of University, Bangalore, India \\ ${ }^{3}$ Dept. Computer Science and Engineering, Visvesvaraya College of Engineering, Bangalore, India \\ *Corresponding Author: savithaphd2016@gmail.com, Tel.: +91-9964157771
}

Available online at: www.isroset.org

Received 30/Jan/2018, Revised 06/Feb/2018, Accepted 18/Feb/2018, Online 28/Feb/2018

\begin{abstract}
Biometric authentication has attracted great interest due to its importance in numerous real-world applications. In this paper, the accuracy issue addressed through multimodal biometric combination. The proposed multimodal biometric combination scheme delivers face, finger print and signature as biometric characteristics, as an input for security purpose. The proposed methodology incorporates Wiener filter for preprocessing the acquired images and Discrete Wavelet Transform (DWT) was used for achieving feature subsets. Then, Linear Discriminant Regression Classification (LDRC) was designed with the combination of Selective Small Reconstruction Error (SSRE), which helps to select the appropriate classes. In experimental analysis, the proposed approach improves the authentication rate by means of False Acceptance Rate (FAR), False Rejection Rate (FRR) and Equal Error Rate (EER). The experimental outcome shows that the proposed methodology improved accuracy in biometric authentication rate up to 5-10\% compared to the existing method: Linear Regression Classification (LRC).
\end{abstract}

Keywords - Biometric Authentication; Discrete Wavelet Transform; Selective Small Reconstruction Error; Wiener Filter;

\section{INTRODUCTION}

Biometric authentication has gained a lot of interest, because of the extensive range of data security and access control. In recent years, the biometric authentication has superior enhancement in dependability \& precision. Some biometric systems offer good person identification performance [1], [2]. However, the most progressive biometric systems are facing several issues, due to the inheritance of data and methodology. Hence, it is not possible to achieve a higher identification rate and attempting to improve the performance of single matchers. Generally, biometric systems functioning on a single biometric feature have several boundaries like Distress with data sensors, Distinctiveness ability, and Lack of universality [3].

In spite of a substantial research work, uni-modal biometric systems are regularly suffering from different types of drawbacks such as non-universal biometric traits, susceptibility to inadequate accuracy because of noisy data [4], [5]. Therefore, uni-modal biometric systems might not be capable of achieving the expected performance requirement in real-world usage [6], [7]. In recent times, this accuracy issue has been addressed through multimodal biometric (multi-biometric) fusion, which integrates the information, by individual biometric. Multimodal biometric fusion has been presented in many past research empirically to enhance the accuracy of biometrics based verification \& identification [8], [9], [10]. Multimodal biometric systems are utilized over physiological or behavioral feature for verification.

Multimodal system utilizes the behavioral characteristics for the verification, identification and combines the several evidence by using the multi-source information [11], [12]. For instance, face recognition, fingerprint, and signature sorts to different at a time. The level of fusion \& integration strategies that can be adopted group of information. In addition, various levels of fusion strategies are utilized such as sensor level fusion, feature level fusion, matching score level, decision level fusion [13], [14], [15], [21]. Its evidence \& fusion to improve the overall decision accuracy \& performance. It mainly used for reducing the false acceptance ratio \& false rejection ratio.

This paper is composed as follows. Section II survey several recent papers on biometric authentication. In section III, an effective unsupervised method (LDRC) is presented with selective small reconstruction error. Section IV shows 
comparative experimental result for existing and proposed strategies. The conclusion is made in Section V.

\section{LiteratURE REVIEW}

Here, we surveyed some of the current investigates concentrated on multi-model biometric authentication methods.

A. Muthukumar et al. [16] have evaluated a multimodal biometric system utilizing Particle Swarm Optimization (PSO). This paper has focused on the security of biometric system, because co-operated biometric patterns cannot be rescinded and also this paper has proposed a multimodal system based on an evolutionary algorithm, PSO that adapts for varying security environments. With these two concerns, this paper had developed a design incorporating adaptability, authenticity and security.

Yuwu et al. [17] have presented a Linear Regression (LR) scheme for solving the solution for LR equation using least square algorithm. Kernel Linear Discriminant Classification (KLRC) is a non-linear extension of LRC, it helps to determine the kernel function. They have employed on three standard databases under some assessment protocols. This methodology not only out-performs the LRC and also it achieves a better performance than typical kernel approaches.

S. M. Huang [18] have presented a LR classification procedure with the help of class-specific exemplification where it was renowned by Between-Class Reconstruction Error (BCRE) and Within Class Recreation Error (WCRE) to discover a discriminant subspace by expanding the estimation of BCRE and diminishing the estimation of WCRE simultaneously. The Main disadvantage of LDRC was expansion of the general BCRE was just dominated by some substantial class-particular BCRE. This thing makes the resulting LRC inaccurate.

B. Ma et al. [19] have illustrated a watermarking-based twostage authentication system for securing the biometric data. In the initial phase of authentication, the credibility of input data was recognized through investigation the validity of extracted patterns. Because of the particular feature of face watermarks, the face detection based classification approaches were obtained for reliable watermark verification in place previous correlation based watermark detection. If authentic, the face patterns can additional assist as extra identity information to enable sub-sequential biometric authentication. Their proposed method's one chief difficult was to assure the robustness during maintaining the discerning individualities of host fingerprints.

H. Saevanee et al. [20] have illustrated a text-based multimodal biometric scheme by exploiting linguistic examination, keystroke dynamics \& behavioral profiling. For evolving an authentication mechanism that can give a less costly, non-intrusive $\&$ uninterrupted solution to the difficult of user authentication. The system was designed by employing a variety of single and multimodal biometric techniques without any additional hardware. The users can benefit from the framework with regards to both device security and convenience of use. The consequences of the simulation evidently displayed that the proposed authentication framework could give continuous and transparent authentication to protect mobile devices.

\section{Proposed Methodology For Biometric}

\section{AUTHENTICATION}

In this stage, images are converted from various sizes to standard size $(32 * 32)$. Subsequently the standard size images are subjected to the noise removal process with the aid of filters. However, during the noise removal process such as median filter and Gaussian filter, the high dimensional information will become a loss. To solve this issue in this paper, we plan to use wiener filter, that minimizes the mean square error and support Image Quality Assessment for fake imageries detection.

\section{A. Wiener Filter}

Wiener filter is employed to eliminate the noise from images based on statistical method. This filter is characterized by a conjecture that image noise is stationary linear random processes and their spectral features are determined. The functioning is further extent using Minimum Mean-Square Error.

The Wiener filter is implemented as represented in the following equation (1) and (2).

$$
\begin{aligned}
& W_{1}(i, j)=\frac{\mathrm{D} *(\mathrm{i}, \mathrm{j}) \mathrm{p}_{\mathrm{s}}(i, j)}{|D(i, j)|^{2} \mathrm{p}_{\mathrm{s}}(i, j)+\mathrm{p}_{\mathrm{n}}(i, j)} \\
& W_{2}(i, j)=\frac{\mathrm{D}^{*}(\mathrm{i}, \mathrm{j}) \mathrm{p}_{\mathrm{s}}(i, j)}{|D(i, j)|^{2} \mathrm{p}_{\mathrm{s}}(i, j)+\mathrm{p}_{\mathrm{n}}(i, j)}
\end{aligned}
$$

Dividing through by $\mathrm{p}_{\mathrm{s}}$,

$$
W(i, j)=\frac{\mathrm{D}^{*}(\mathrm{i}, \mathrm{j})}{|D(i, j)|^{2}+\frac{\mathrm{p}_{\mathrm{n}}(i, j)}{p_{s}(i, j)}}
$$


Where,

$W_{1}(i, j)=$ wiener filter for noise removal in fingerprint image

$W_{2}(i, j)=$ wiener filter for noise removal in face image

$D(i, j)=$ Degradation Function $(D F)$ on image

$D^{*}(\mathrm{i}, \mathrm{j})=$ Complex conjugate of DF on image

$\mathrm{p}_{\mathrm{n}}(i, j)=$ Power Spectral Density (PSD) of Noise on image

$\mathrm{p}_{\mathrm{s}}(i, j)=$ PSD of un-degraded image

\section{Discrete WAVElet Transform}

To explain the fraudulent attack and to build the superior biometric authentication structure, each of the image fusion procedure merges the two or more images into a single image. In order to make the fusion of images initially, every image is converted to wavelet representation to remap an image without any information loss based on DWT which makes the picture noticeably practical for image fusion. Successively, wavelet coefficients are fused based on the cultivated fusion rule of wavelet transform domain. The fused images are constructed based on inverse DWT.

\section{PRINCIPLE Component ANALYSIS (PCA)}

Here, PCA based feature extraction is exploited to extract the global features from multimodal images. Moreover, PCA is utilized to decrease the dimensionality of an image. Initially the $2 \mathrm{D}$ images are converted into $1 \mathrm{D}$ images and indicated as $1 \times i j$ by merging the pixels in the original image. Then, subtract the row wise mean from each dimension and place the result in matrix $\mathrm{R}$. Construct the $j \times j$ covariance matrix for building the matrix become square, which is used for calculation of Eigen vectors and Eigen values. Construction of a covariance matrix $j \times j$ by equation (4).

$$
R^{\prime} \times R
$$

Find the eigenvalues and eigenvectors of the images by solving equation (5),

$$
(R-\lambda) V=0
$$

Where, $\lambda$ is an Eigen value and $V$ is an eigenvector. This modules are not effective, which are eliminated in order to diminish the dimensionality. Thus, selecting the first $v$ Eigen vectors $(v<j)$. By considering the selected $v$ Eigen and feature vectors are calculated and then build the matrix with Eigen vectors as columns represents in equation (6).

$$
F=\left(e i g_{1}, e i g_{2}, \cdots, e i g_{j}\right)
$$

Transpose vector is under taken after the feature vector $F$ is constructed and then multiply the transpose vector with the original data set.

\section{Proposed Linear Discriminant Regression Classification}

LRC scheme is enhanced by utilizing a LDRC algorithm through absorbing fisher criterion into LRC that prompts to increase the proportion of the BCRE over the WCRE. Through employing discriminant investigation, we need to separate the patterns of various classes by picking the projection directions on which the patterns of different classes are far from each other while keeping the patterns of the same classes be as close to each other as possible. Therefore, the proposed LDRC could estimate an optimum prediction in such a way that the ratio of the BCRE over the WCRE achieved by the LRC is maximized. An ideal projection matrix is found to extend the original picture to a more segregating low-dimensional space. In the new lowdimensional space, LDRC can achieve better recognition performance.

Generally, classification error happens when the true class and false class have similar small reconstruction errors. So, if only the classes with small reconstruction errors are considered when evaluating BCRE, the reconstruction error difference between the true class and false class will be increased. This helps to correctly classify the probe image. In LDRC, when calculating the BCRE value, all other classes are considered except the class from which the probe image comes from. In our proposed methodology, we propose a method based on SSRE that takes the classes with a small reconstruction error rather than use all those classes for calculating the BCRE. The reason is that when classifying the probe image, we only care about the classes with small reconstruction error. Those classes with large reconstruction error are easily excluded out.

\section{A. Linear Regression Classification (LRC)}

Consider $S$ subjects with $g_{i}$ training images from the $i^{\text {th }}$ class where $1 \leq i \leq S$. Through the projection of the image space into the multimodal space, the matrix $F$ contains feature vectors from $S$ subjects and $F=\left[F_{1}, \ldots, F_{i} \ldots, F_{S}\right]$. In order to apply regression analysis to estimate class specific 
model and grouping the column vectors $f_{i, j}$ regarding the class-membership $i^{\text {th }}$ class represents as equation (7),

$$
F_{i}=\left[f_{i, 1}, \ldots, f_{i, j} \ldots, f_{i, g_{i}}\right] \in \mathfrak{R}^{L^{*} g_{i}}
$$

Where the vector $f_{i, j}$ is a column vector in size of $\mathrm{L}^{*} 1$. Thus, in the training stage, the $i^{\text {th }}$ class is represented by a vector space $F_{i}$, represents the predictor for each subject.

If $d$ belongs to the $i^{\text {th }}$ class, which represented as a linear combination of the training pictures from the $i^{\text {th }}$ class and it defined as equation (8).

$$
y=F_{i} \beta_{i}+e, \mathrm{i}=1,2 \ldots \mathrm{S}
$$

Where $\beta_{i} \in \mathfrak{R}^{g_{i}{ }^{* l}}$ is the vector of regression parameters and $e$ is an error vector that is an i.i.d. random variable with zero mean and variance $\sigma^{2}$. Major aim of the linear regression is to obtain $\hat{\beta}_{i}$ to minimize the residual errors as given in equation (9).

$$
\hat{\beta}_{i}=\underset{\beta_{i}}{\arg \min }\left\|F_{i} \beta_{i}-d\right\|_{2}^{2}, \mathrm{i}=1,2 \ldots \mathrm{S}
$$

The estimate of the regression parameter vectors can be computed through equation (10).

$$
\hat{\beta}_{i}=\left(F_{i}^{T} F_{i}\right)^{-1} F_{i}^{T} d
$$

The estimated parameters $\hat{\beta}_{i}$ and predictors $F_{i}$, used to predict the response vector $\hat{d}_{i}$ for the $i^{\text {th }}$ class as equation (11).

$$
\hat{d}_{i}=F_{i} \hat{\beta}_{i}, \quad \mathrm{i}=1,2 \ldots, \mathrm{S}
$$

By substituting (10) into (11), by using following equation (12).

$$
\hat{d}_{i}=F_{i}\left(F_{i}^{T} F_{i}\right)^{-1} F_{i}^{T} d, \mathrm{i}=1,2 \ldots, \mathrm{S}
$$

Therefore, class specific projection expressed in equation (13).

$$
\hat{d}_{i}=H_{i} d, \mathrm{i}=1,2 \ldots, \mathrm{S}
$$

Where $\hat{d}_{i}$ is the projection of $\mathrm{d}$ onto the subspace of the $i^{\text {th }}$ class by the class projection matrix $H_{i}=F_{i}\left(F_{i}^{T} F_{i}\right)^{-1} F_{i}^{T}$. Here the projection matrix $\left(H_{i}\right)$ is a symmetric matrix and also idempotent.

The linear regression actually based on the minimum distance between the original vector and the projected vector.

The predicted vector $\hat{d}_{i}$ will be the closest vector to the original vector. The Euclidean distance measure between the predicted response vectors and the original vector by using $i^{*}$ given in equation (14).

$$
i^{*}=\underset{i}{\arg \min }\left\|\hat{d}_{i}-d\right\|, \mathrm{i}=1,2 \ldots, \mathrm{S}
$$

\section{B. Linear Discriminant Regression Classification}

Arithmetically, a set of $\mathrm{M}$ training images is given and each gray scale training pictures is in size of $a^{*} b$ and is represented as $v_{m} \in \mathfrak{R}^{a^{*} b} \mathrm{~m}=1,2 \ldots$, M. Each image is transformed to a column vector $x_{m} \in \mathfrak{R}^{L^{* 1}}$, Where $L=a * b$. By load up all $x_{m}$, the collected data is given as $\mathrm{X}=$ $\left[x_{1}, \ldots, x_{m}, \ldots, x_{M}\right] \in \mathfrak{R}^{L^{*} M}$ and the corresponding class label of $x_{m}$ is denoted as $l\left(x_{m} \in\{1,2, \ldots, c\}\right)$, where $\mathrm{c}$ is the total number of classes.

\section{Linear Discriminant Regression Analysis}

The proposed LDRC method formulation helps to find the optimization problem to maximize the objective function given as in the equation (15).

$$
\max _{U} J(U)=\max _{U}\left(\frac{E_{B C}}{E_{W C}}\right),
$$

Where, $\mathrm{U}$ is the optimal projection matrix and $E_{B C}, E_{W C}$ denote between-class and within-class reconstruction errors from LRC, respectively. Hence, the proposed method aims at finding an optimal mapping $U=\left[u_{1}, \ldots, u_{n}, \ldots, u_{q}\right]$ from the original space $x_{m}$ to the subspace $y_{m}=U^{T} x_{m}$ that is effective for linear regression classification. The samples from specific 
object classes known to recline on a linear subspace is used to improve the LDRC algorithm that noted by using $l\left(x_{m}\right)=l\left(y_{m}\right)$. So the feature vectors are used to estimate the class-specific projection matrix $H_{i}^{i j}$ for each class $i$. The objective function expressed in equation (16).

$$
J(U)=\frac{E_{B C}}{E_{W C}}=\frac{\frac{1}{M(c-1)} \sum_{i=1}^{M} \sum_{j=1, j \neq l\left(\hat{x}_{i}\right)}^{c}\left\|d_{i}-\hat{d}_{i j}^{\text {inter }}\right\|^{2}}{\frac{1}{M} \sum_{i=1}^{M}\left\|d_{i}-\hat{d}_{i}^{\text {int } r a}\right\|^{2}}
$$

$$
\wedge \text { int } e r
$$

Where, $\hat{d}_{i j}^{\text {int } e r}=H_{j}^{d} d_{i} \quad$ denotes the inter-class projection of $d_{i}$ by the LRC in (13) with the patterns from the different $j^{\text {th }}$ class, (i.e., $j \neq l\left(d_{i}\right)$ and $\wedge$ int $r a$

$d_{i j}=H_{i}^{d} d_{i}$ denotes the intra-class projection of $d_{i}$ by the LRC in (13) with the remaining patterns in the same class (i.e., $i=l\left(d_{i}\right)$ represents in equation (17),

$$
\begin{aligned}
& J(U)=\frac{\frac{1}{M(c-1)} \sum_{i=1}^{M} \sum_{j=1, j \neq l\left(\hat{x}_{i}\right)}^{c}\left\|d_{i}-H_{j}^{d} d_{i}\right\|^{2}}{\frac{1}{M} \sum_{i=1}^{M}\left\|d_{i}-H_{i}^{y} d_{i}\right\|^{2}} \\
& =\frac{\frac{1}{M(c-1)} \sum_{i=1}^{M} \sum_{j=1, j \neq l\left(\hat{x_{i}}\right)}^{c}\left\|U^{T} x_{i}-H_{j}^{d} U^{T} x_{i}\right\|^{2}}{\frac{1}{M} \sum_{i=1}^{M}\left\|U^{T} x_{i}-H_{j}^{d} U^{T} x_{i}\right\|^{2}}
\end{aligned}
$$

With some algebraic deduction, equation (17) can be rewritten as (18).

$$
\begin{gathered}
J(U)=\frac{\frac{1}{M(c-1)} \sum_{i=1}^{M} \sum_{j=1, j \neq l\left(\hat{x}_{i}\right)}^{c} \operatorname{tr}\left[U^{T}\left(x_{i}-x_{i j}^{\mathrm{int} e r}\right)\left(x_{i}-x_{i j}^{\mathrm{int} e r}\right)^{T} U\right]}{\frac{1}{M} \sum_{i=1}^{M} \operatorname{tr}\left[U^{T}\left(x_{i}-x_{i j}^{\mathrm{int} e r}\right)\left(x_{i}-x_{i j}^{\mathrm{int} e r}\right)^{T} U\right]} \\
=\frac{\operatorname{tr}\left(U^{T} E_{b} U\right)}{\operatorname{tr}\left(U^{T} E_{w} U\right)}
\end{gathered}
$$

Where, value of $E_{b}$ and $E_{w}$ is given by equation (19) and (20) respectively.

$$
\begin{gathered}
E_{b}=\frac{1}{M(c-1)} \sum_{i=1}^{M} \sum_{j=1, j \neq l\left(\hat{x}_{i}\right)}^{c}\left(x_{i}-x_{i j}^{\mathrm{int} e r}\right)\left(x_{i}-x_{i j}^{\mathrm{int} e r}\right)^{T} \\
E_{w}=\frac{1}{M} \sum_{i=1}^{M}\left(x_{i}-x_{i j}^{\mathrm{int} e r}\right)\left(x_{i}-x_{i j}^{\mathrm{int} e r}\right)^{T}
\end{gathered}
$$

$E_{b}$ And $E_{w}$ denotes inter-class and intra-class reconstruction error matrices achieved by the LRC, respectively. Finally, the objective function depicted in (15) can be expressed in equation (21).

$$
\max _{U} J(U)=\underset{U}{\arg \max } \frac{U^{T} E_{b} U}{U^{T} E_{w} U}
$$

In addition, LRC is usually much smaller than the dimensionality of the sample space that number of samples per class for computing the intra-class reconstruction error. As a result to address this problem is called "small sample size" (SSS) problem, so the term $\varepsilon I$ is added without affecting the subspace. The objective function stated in (21) then becomes equation (22).

$$
\underset{U}{\arg \max } J(U)=\underset{U}{\arg \max } \frac{U^{T} E_{b} U}{U^{T}\left(E_{w}+\varepsilon I\right) U},
$$

Where, $\varepsilon$ is a small positive number and $\mathrm{I}$ is an identity matrix of proper size. Equation (22) can be solved by maximizing the numerator while keeping the denominator constant, so (22) can be reformulated as the following constrained optimization problem represented in equation (23),

$\underset{U}{\arg \max } U^{T} E_{b} U$

$$
\text { s.t } U^{T}\left(E_{w}+\varepsilon I\right) U=k
$$

Where, $\mathrm{k}$ is a constant. Equation (23) can be accomplished by using Lagrange multipliers. Therefore, the transformation matrix $U=\left[u_{1}, \ldots, u_{n}, \ldots, u_{q}\right]$ are maximizes the objective function that obtained by solving the generalized eigenvalue problem as given in the equation (24).

$$
E_{b} u_{k}=\lambda_{k}\left(E_{w}+\varepsilon I\right) u_{k}, \mathrm{k}=1,2, \ldots, \mathrm{q},
$$

Where $\lambda_{l} \geq \ldots \geq \lambda_{k} \ldots \geq \lambda_{d}$.

$E_{b} u_{k}=\lambda_{k}\left(E_{w}+\varepsilon I\right) u_{k}$, is eigenvalue of the proposed LDRC, which is used to evaluate the effectiveness of the multimodal 
image. After classification, measures rate of FAR and FRR for calculates the total performance. By using training image is greater than testing image, which help to improve the accuracy and performance.

\section{EXPERIMENTAL SET UP}

In this section, we evaluate our proposed LDRC multi-model methodology by using Unimodel methodology. Thus, in this research we have considered standard benchmark ORL face dataset and CASIA fingerprint dataset. Here we have taken 40 persons considered and for each person 10 images of face and fingerprint are taken for implementation. We evaluate our proposed methodology in terms of FAR, FRR and accuracy. The ORL face dataset has distinct face images of a person differentiated by various factors such as lightening, pose and expressions. The CASIA fingerprint dataset has distinct images of a person differentiated by different angles of left and right hand images.

\section{A. Factors for Comparative Analysis}

The brief notes for the factors used in comparative analysis is discussed in this section.

\section{False Acceptance Rate (FAR)}

It is used to measure of a biometric security system that will wrongly grant an access effort made by an unauthorized user.

FAR $=\frac{\text { Number of Falsely Accepted images }}{\text { Total number of persons out of database }}$

\section{False Rejection Rate (FRR)}

It is used to measure of biometric security system that will wrongly reject an access effort made by an authorized user.

$$
\text { FRR }=\frac{\text { Total number of persons in the databese }}{\text { Number of Falsely rejected images }}
$$

Using this formulas, to calculates FAR, FRR for increasing successive rate of verification and identification.

\section{Equal Error Rate (EER)}

The rate at which both acceptance and rejection errors are equivalent. The value of the EER can be effortlessly gained from the ROC curve. Generally, the device with the least EER is the most accurate.

\section{B. Comparative Analysis of Accuracy}

In this section, we evaluate our proposed LDRC methodology by comparing with LRC in terms of accuracy.
We obtain values of accuracy for LRC and LDRC for various values of dimensions. The following figure 1 indicates the comparative analysis of fusion image accuracy for the combination of face and finger. The figure 2 represents the accuracy of the LRC and LDRC for face images and the figure 3 depicts that evaluation of accuracy of the fingerprint images. Figure 4 indicates the comparative analysis of fusion image accuracy for the combination of face and signature and the figure 5 depicts that evaluation of accuracy of the signature images. By analyzing the figures 1, 2, 3, 4 and 5, it can be said that our proposed LDRC methodology achieves better results than the LRC methodology. The reason behind this is our proposed SSRE concept is incorporated with LDRC, which only considers the classes with small reconstruction error when calculating BCRE, the reconstruction error difference between the true class and false class will be increased which leads to increase the accuracy of classification.

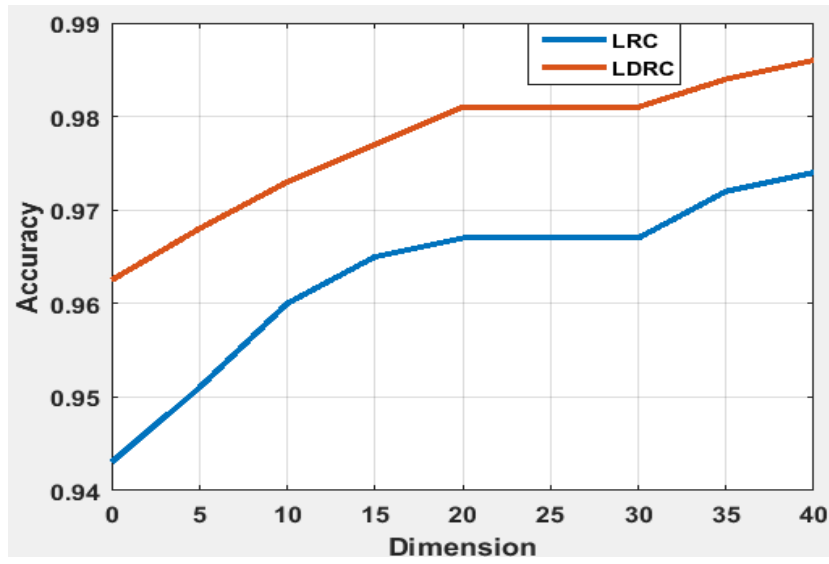

Figure 1. Fusion image 60(Face and Finger) train

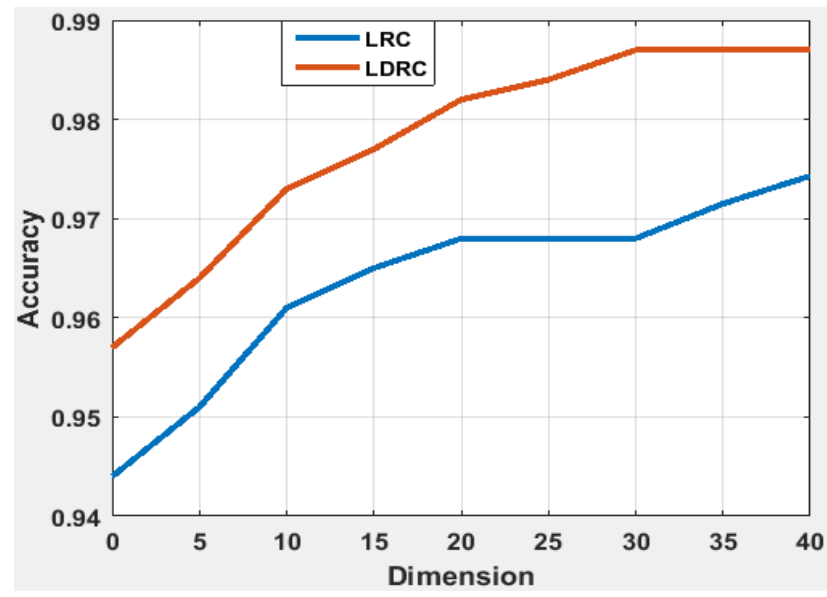

Figure 2. Face image 60 train 


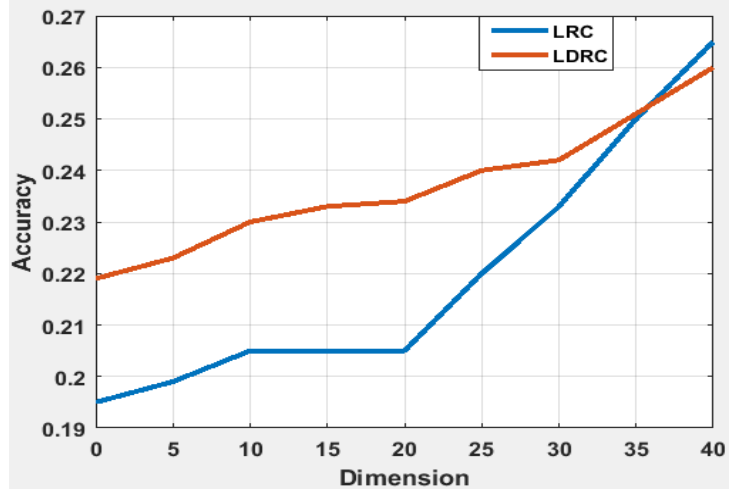

Figure 3. Finger image 60 train

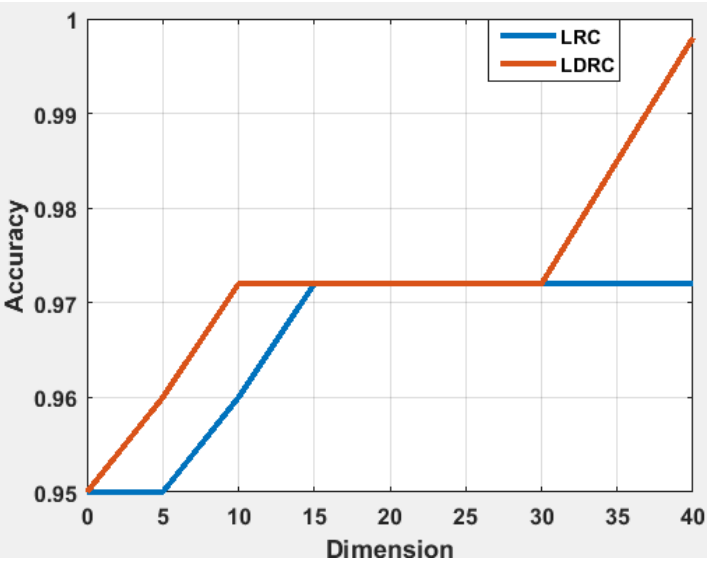

Figure 4. Fusion image 60(Face and Signature) train

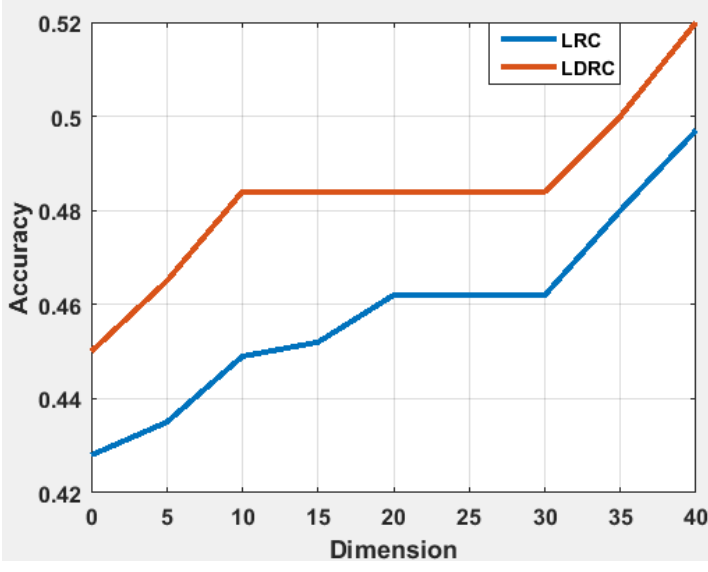

Figure 5. Signature image 60 train

\section{Comparative Analysis of FRR and FAR}

In this section, we evaluate our proposed LDRC methodology by comparing with LRC in terms of FRR, FAR and EER based on varying the threshold values. The following figure 6 indicates the comparative analysis of fusion image error for the combination of face and finger. The figure 7 represents the error factors of the LRC and
LDRC for face images and the figure 8 depicts that evaluation of error factors for the fingerprint images. When the threshold value becomes same for more than one class, the threshold value of selected class will be decreased to differentiate the threshold values for proper classification. If the threshold value is less FAR value will be increased and if the threshold value is high, then the FRR value will be increased which is stated in the following figure 6 and 7 . The selection of proper threshold determined by the EER and it leads to attain the better classification accuracy. Selection of the EER is determined by the values of FAR and FRR, which is, represented in the figure 6 and 7 for fusion images and face images respectively. For the fingerprint images, the selection of the EER is not found because of the dimensions of fingerprint images are improper stated in figure 8, moreover, this is the main reason for leading us to make the proposed fusion methodology. Figure 9 represents the unimodal signature image and the figure 10 indicates the comparative analysis of fusion image error for the combination of face and signature.

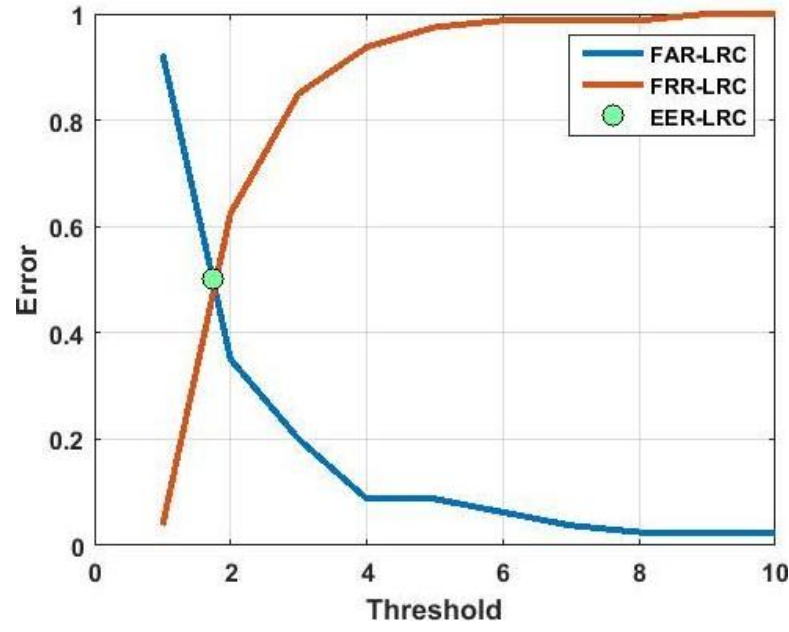

Figure 6. Selection of EER for fusion images (Face and finger)

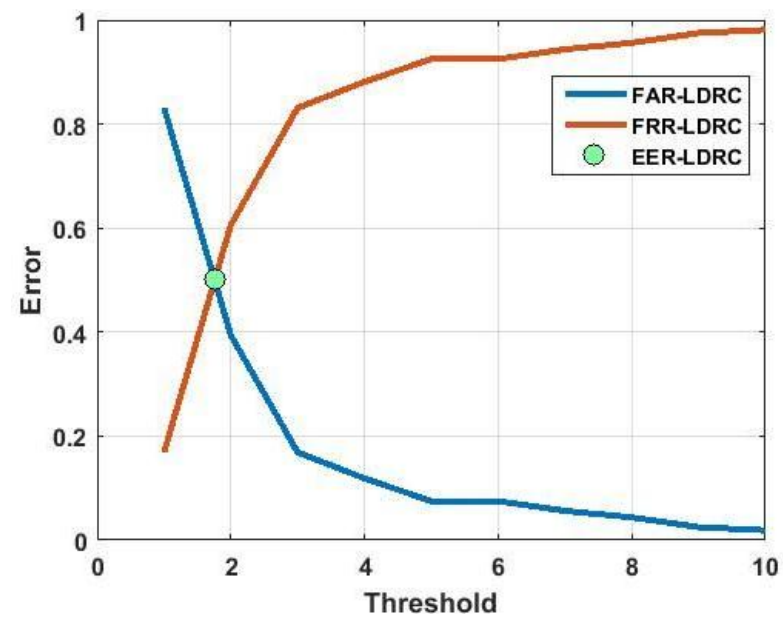

Figure 7. Selection of EER for face images 


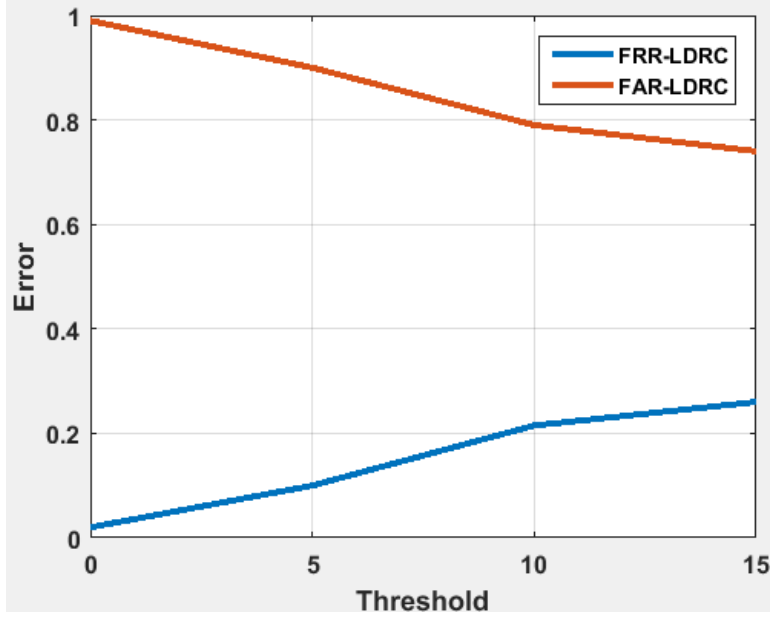

Figure 8. Selection of EER for fingerprint images

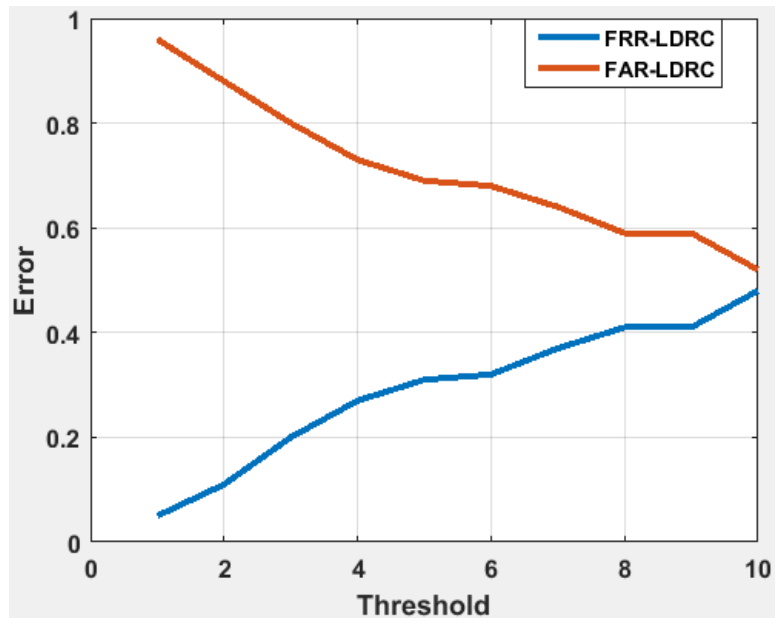

Figure 9. Unimodal signature image

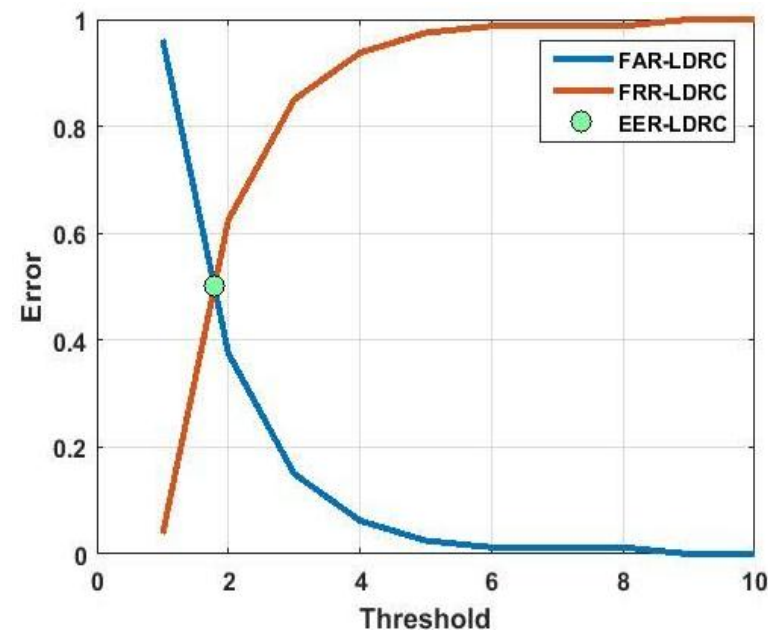

Figure 10. Selection of EER for fusion images (Face and signature)

\section{Comparative Analysis for Various Training Data}

The table 1 describes the accuracy of proposed LDRC and LRC for various training data for the combination of face and finger. By analyzing the table 1, our proposed LDRC methodology performed better than the existing LRC method in terms of accuracy for multi-model images and Unimodel images.

Table 1. Represents the accuracy of LDRC and LRC for the combination of face and finger

\begin{tabular}{|c|c|c|c|c|c|c|c|c|c|}
\hline \multicolumn{1}{|c|}{ Multi Model } & \multicolumn{7}{c|}{ Unimodel } \\
\hline \multirow{3}{*}{$\begin{array}{c}\text { Meth } \\
\text { ods }\end{array}$} & \multicolumn{3}{|c|}{$\begin{array}{c}\text { Fusion (Face and } \\
\text { fingerprint) }\end{array}$} & \multicolumn{3}{c|}{ Face } & \multicolumn{3}{c|}{ Fingerprint } \\
\cline { 2 - 10 } & $\mathbf{6 0}$ & $\mathbf{4 0}$ & $\mathbf{2 0}$ & $\mathbf{6 0}$ & $\mathbf{4 0}$ & $\mathbf{2 0}$ & $\mathbf{6 0}$ & $\mathbf{4 0}$ & $\mathbf{2 0}$ \\
& Trai & Train & Tra & Tra & Tra & Tra & Tra & Tra & Tra \\
& $\boldsymbol{n}$ & & in & in & in & in & in & in & in \\
\hline LDR & 98.8 & \multirow{2}{*}{94.16} & 85. & 98. & 93. & 84. & 26. & 0.1 & 0.1 \\
C & 5 & & 14 & 88 & 81 & 44 & 3 & 99 & 537 \\
\hline \multirow{2}{*}{ LRC } & 97.5 & \multirow{2}{*}{90.41} & 75. & 97. & 92. & 82. & 26. & 0.1 & 0.1 \\
& 0 & & 71 & 57 & 05 & 90 & 9 & 675 & 387 \\
\hline
\end{tabular}

Table 2 labels the accuracy of proposed LDRC and LRC for various training data for the combination of face and signature. By analyzing the table 2, our proposed LDRC methodology performed better than the existing LRC method. Compared to the combination of (face and finger print), the combination of (face and signature) shows an effective improvement in terms of accuracy for multi-model images and Unimodel images.

Table 2. Represents the accuracy of LDRC and LRC for the combination of face and signature

\begin{tabular}{|c|c|c|c|c|c|c|c|c|c|}
\hline \multicolumn{10}{|c|}{ Accuracy } \\
\hline \multirow{3}{*}{$\begin{array}{c}\text { Meth } \\
\text { ods }\end{array}$} & \multirow{2}{*}{\multicolumn{3}{|c|}{$\begin{array}{c}\text { Multi Model } \\
\text { Fusion (Face and } \\
\text { signature) }\end{array}$}} & \multicolumn{6}{|c|}{ Unimodel } \\
\hline & & & & \multicolumn{3}{|c|}{ Face } & \multicolumn{3}{|c|}{ Signature } \\
\hline & $\begin{array}{c}60 \\
\text { Trai } \\
n\end{array}$ & $\begin{array}{c}40 \\
\text { Train }\end{array}$ & $\begin{array}{c}20 \\
\text { Tra } \\
\text { in }\end{array}$ & $\begin{array}{c}60 \\
\text { Tra } \\
\text { in }\end{array}$ & $\begin{array}{c}40 \\
\text { Tra } \\
\text { in }\end{array}$ & $\begin{array}{c}20 \\
\text { Tra } \\
\text { in }\end{array}$ & $\begin{array}{c}60 \\
\text { Tra } \\
\text { in }\end{array}$ & $\begin{array}{c}40 \\
\text { Tra } \\
\text { in }\end{array}$ & $\begin{array}{c}20 \\
\text { Tra } \\
\text { in }\end{array}$ \\
\hline $\begin{array}{l}\text { LDR } \\
\mathrm{C}\end{array}$ & 99.9 & 97.90 & $\begin{array}{l}94 . \\
43\end{array}$ & $\begin{array}{l}98 . \\
88 \\
\end{array}$ & $\begin{array}{l}93 . \\
81\end{array}$ & $\begin{array}{l}84 . \\
44\end{array}$ & $\begin{array}{l}53 . \\
1 \\
\end{array}$ & $\begin{array}{l}51 . \\
02\end{array}$ & $\begin{array}{l}48 . \\
97 \\
\end{array}$ \\
\hline LRC & $\begin{array}{l}97.7 \\
5\end{array}$ & 96.78 & $\begin{array}{l}93 . \\
82\end{array}$ & $\begin{array}{l}97 . \\
57\end{array}$ & $\begin{array}{l}92 . \\
05\end{array}$ & $\begin{array}{l}82 . \\
90\end{array}$ & 50 & $\begin{array}{l}48 . \\
05\end{array}$ & $\begin{array}{l}46 . \\
89\end{array}$ \\
\hline
\end{tabular}

Table 3 represents the values of FAR, FRR, EER of proposed LDRC and LRC for the combination of face and fingerprint. Our proposed LDRC methodology performed better than the existing LRC method in terms of FAR, FRR and EER for multi-model images and Unimodel images. 
Table 3. Depicts values of FAR, FRR, EER of LDRC and LRC for the combination of face and finger

\begin{tabular}{|c|l|l|l|l|l|l|l|l|l|}
\hline \multirow{3}{*}{$\begin{array}{c}\text { Method } \\
\text { S }\end{array}$} & \multicolumn{3}{|c|}{$\begin{array}{c}\text { Multi Model } \\
\text { fingerprint) }\end{array}$} & \multicolumn{4}{c|}{ Face } & \multicolumn{3}{c|}{ Fingerprint } \\
\cline { 2 - 9 } & FAR & $\boldsymbol{F R R}$ & $\begin{array}{c}\boldsymbol{E E} \\
\boldsymbol{R}\end{array}$ & $\begin{array}{c}\boldsymbol{F A} \\
\boldsymbol{R}\end{array}$ & $\begin{array}{c}\boldsymbol{F R} \\
\boldsymbol{R}\end{array}$ & $\begin{array}{c}\boldsymbol{E E} \\
\boldsymbol{R}\end{array}$ & $\begin{array}{c}\boldsymbol{F A} \\
\boldsymbol{R}\end{array}$ & $\begin{array}{c}\boldsymbol{F R} \\
\boldsymbol{R}\end{array}$ & $\begin{array}{c}\boldsymbol{E E} \\
\boldsymbol{R}\end{array}$ \\
\hline \multirow{3}{*}{ LDRC } & $\begin{array}{l}0.15 \\
58\end{array}$ & $\begin{array}{l}0.844 \\
1\end{array}$ & $\begin{array}{l}1.7 \\
09\end{array}$ & $\begin{array}{l}0.1 \\
7\end{array}$ & $\begin{array}{l}0.8 \\
52\end{array}$ & $\begin{array}{l}1.6 \\
80 \\
5\end{array}$ & $\begin{array}{l}0.8 \\
30\end{array}$ & $\begin{array}{l}0.1 \\
70\end{array}$ & 0 \\
\hline \multirow{2}{*}{ LRC } & $\begin{array}{l}0.19 \\
95\end{array}$ & 0.800 & $\begin{array}{l}1.9 \\
30 \\
2\end{array}$ & $\begin{array}{l}0.2 \\
00\end{array}$ & $\begin{array}{l}0.7 \\
99\end{array}$ & $\begin{array}{l}1.8 \\
90 \\
1\end{array}$ & $\begin{array}{l}0.8 \\
60\end{array}$ & $\begin{array}{l}0.1 \\
38\end{array}$ & 0 \\
\hline
\end{tabular}

Table 4 signifies the values of FAR, FRR, EER of proposed LDRC and LRC for the combination of face and signature. Our proposed LDRC methodology performed better than the existing LRC method. Compared to the combination of (face and finger print), the combination of (face and signature) shows an effective improvement in terms of FAR, FRR and EER for multi-model images and Unimodel images.

Table 4. Depicts values of FAR, FRR, EER of LDRC and LRC for the combination of face and signature

\begin{tabular}{|c|c|c|c|c|c|c|c|c|c|}
\hline \multirow{3}{*}{$\begin{array}{c}\text { Metho } \\
\quad d s\end{array}$} & \multirow{2}{*}{\multicolumn{3}{|c|}{$\begin{array}{c}\text { Multi Model } \\
\begin{array}{c}\text { Fusion (Face and } \\
\text { signature) }\end{array}\end{array}$}} & \multicolumn{6}{|c|}{ Unimodel } \\
\hline & & & & \multicolumn{3}{|c|}{ Face } & \multicolumn{3}{|c|}{ Signature } \\
\hline & $F A R$ & $F R R$ & $\begin{array}{c}E E \\
R\end{array}$ & $\begin{array}{c}F A \\
R\end{array}$ & $\begin{array}{c}F R \\
R\end{array}$ & $\begin{array}{c}E E \\
R\end{array}$ & $\begin{array}{c}F A \\
R\end{array}$ & $\begin{array}{c}F R \\
R\end{array}$ & $\begin{array}{c}E E \\
R\end{array}$ \\
\hline$L D R C$ & $\begin{array}{l}0.13 \\
9\end{array}$ & 0.83 & $\begin{array}{l}1.4 \\
67\end{array}$ & $\begin{array}{l}0.1 \\
48\end{array}$ & $\begin{array}{l}0.8 \\
52\end{array}$ & $\begin{array}{l}1.6 \\
80 \\
5\end{array}$ & $\begin{array}{l}0.8 \\
76\end{array}$ & $\begin{array}{l}0.1 \\
8\end{array}$ & 0 \\
\hline$L R C$ & $\begin{array}{l}0.18 \\
9\end{array}$ & 0.768 & $\begin{array}{l}1.6 \\
73\end{array}$ & $\begin{array}{l}0.2 \\
00\end{array}$ & $\begin{array}{l}0.7 \\
99\end{array}$ & $\begin{array}{l}1.8 \\
90 \\
1\end{array}$ & $\begin{array}{l}0.8 \\
9\end{array}$ & $\begin{array}{l}0.1 \\
46\end{array}$ & 0 \\
\hline
\end{tabular}

\section{CONCLUSION}

This paper evaluates an effective scheme named as linear discriminant regression classification for Unimodel and multi-model images. In this scenario, the experimental analysis was performed on two different combination of biometrics such as (face and finger print) and (face and signature). At first, Weiner filter was used for enhancing the quality of acquired image by eliminating the unwanted noise. Then, calculate the features from pre-processed images using PCA methodology. Based on the selection of set of features, the DWT make the fusion of (face and fingerprint) and (face and signature) images. Associated to other obtainable approaches in biometric authentication, the proposed scheme delivered an effective performance by means of FAR, EER and FRR, and also around 5-10\% of improvement in accuracy than the existing methods. In the future work, for further improving the authentication rate, the descriptor level features were combined with an appropriate multi-objective classification method.

\section{REFERENCES}

[1] A.K. Jain, A. Ross, S. Prabhakar, "An introduction to biometric recognition", IEEE Transactions on Circuits and Systems for Video Technology, Vol.14, No.1, pp.4-20, 2004.

[2] A.K. Jain, A. Ross, "Multibiometric systems", Communications of the ACM, Vol.47, No.1, pp.34-40, 2004.

[3] R. Raghavendra, B. Dorizzi, A. Rao, G.H. Kumar, "Designing efficient fusion schemes for multimodal biometric systems using face and palmprint", Pattern Recognition, Vol.44, No.5, pp.10761088, 2011.

[4] N. Poh, J. Kittler, "A unified framework for biometric expert fusion incorporating quality measures", IEEE Transactions on Pattern Analysis and Machine Intelligence, Vol.34, no.1, pp.3-18, 2012.

[5] M. Hofmann, S.M. Schmidt, A.N. Rajagopalan, G. Rigoll, "Combined face and gait recognition using alpha matte preprocessing", In the Proceedings of 5th IAPR International Conference on Biometrics (ICB), pp.390-395, 2012.

[6] U. Uludag, S. Pankanti, S. Prabhakar, A.K. Jain, "Biometric cryptosystems: issues and challenges", Proceedings of the IEEE, Vol.92, No.6, pp.948-960, 2004.

[7] W.J. Scheirer, T.E. Boult, "Bio-cryptographic protocols with bipartite biotokens," In the Proceedings of Biometrics Symposium, pp.9-16, 2008.

[8] A. Mishra, "Multimodal biometrics it is: need for future systems", International journal of computer applications, Vol.3, No.4, pp.28$33,2010$.

[9] A. Ross, A.K. Jain, "Multimodal biometrics: An overview", In the Proceedings of 12th European Conference In Signal Processing, pp.1221-1224, 2004.

[10] S. Sheena, S. Mathu, "A Study of Multimodal Biometric System", IJRET: International Journal of Research in Engineering and Technology, Vol.3, No.14, pp.93-97, 2014.

[11] K. Sasidhar, V.L. Kakulapati, K. Ramakrishna, K. KailasaRao, "Multimodal biometric systems-Study to improve accuracy and performance", International Journal of Computer Science \& Engineering Survey (IJCSES), Vol.1, No.2, 2010.

[12] M. Soltane, M. Bakhti, "Multi-modal biometric authentications: concept issues and applications strategies", International Journal of Advanced Science and Technology, Vol.48, 2012.

[13] M. Pathak, N. Srinivasu, "Analysis of Multimodal Biometric System Based on Level of Fusion", International Journal of Inventive Engineering and Sciences (IJIES), Vol.3, no.8, 2015.

[14] Y. Li, M. Shi, E. Zhu, J. Yin, J. Zhao, "A Multimodal Fusion Algorithm Based on FRR and FAR Using SVM", International Journal of Security and Its Applications, Vol.7, No.3, pp.65-74, 2013.

[15] G. Savitha, L. Vibha, K.R. Venugopal, "Multimodal Biometric Authentication System Using Ldr Based on Selective Small Reconstruction Error", Journal of Theoretical and Applied Information Technology, Vol.92, No.1, pp.171, 2016.

[16] A. Muthukumar, C. Kasthuri, S. Kannan, "Multimodal Biometric Authentication using Particle Swarm Optimization Algorithm with Fingerprint And Iris", ICTACT Journal on Image and Video Processing, Vol.2, No.3, 2012.

[17] Y. Lu, X. Fang, B. Xie, "Kernel linear regression for face recognition", Neural Computing and Applications, Vol.24, No.7-8, pp.1843-1849, 2014.

[18] S. M. Huang, J. F. Yang, "Linear Discriminant Regression Classification for Face Recognition", IEEE Transactions On Signal Processing Letters, Vol.20, No.1, 2013.

[19] B. Ma, Y. Wang, C. Li, Z. Zhang, D. Huang, "Secure multimodal biometric authentication with wavelet quantization based fingerprint watermarking", Multimedia tools and applications, Vol.72, No.1, pp.637-666, 2014. 
[20] H. Saevanee, N. Clarke, S. Furnell, V. Biscione, "Continuous user authentication using multi-modal biometrics", Computers \& Security, Vol.53, pp.234-246, 2015.

[21] A. Gupta, E. Sharma, N. Sachan, N. Tiwari, "Door Lock System through Face Recognition Using MATLAB”, International Journal of Scientific Research in Computer Science and Engineering, Vol.1, No.3, pp.51-55, 2013.

\section{Authors Profile}

Savitha G. is currently the Associate Professor, Department of Computer Science and Engineering, BNM Institute of Technology, Bangalore. She obtained her Bachelor of Engineering Degree and her Masters degree in Computer Science and Engineering from Visvesvaraya Technological University, Belgaum. She is pursuing her Ph.D. in Computer Science and

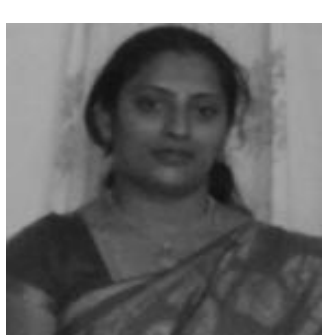
Engineering of Visvesvaraya Technological University under the guidance of Dr. Vibha L, Professor, Department of Computer Science and Engineering, BNMIT, Bangalore. Her research interests include Computer Vision, Pattern Recognition and Biometrics.

Dr. Vibha Lakshmikantha is currently the Professor, Department of Computer Science and Engineering, BNM Institute of Technology, VTU, Bangalore. She obtained her Bachelor of Engineering Degree and her Masters degree in Electronics and Communication from University Visvesvaraya College of Engineering, Bangalore University. She

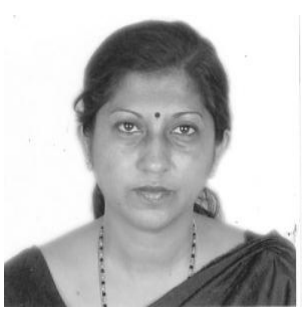
was awarded Ph. D. in Computer Science from Dr. MGR Research and Educational Institute, Chennai, India.

Dr. $K R$ Venugopal is currently the Principal, University Visvesvaraya College of Engineering, Bangalore University, Bangalore. He obtained his Bachelor of Engineering from University Visvesvaraya College of Engineering. He received his Masters degree in Computer Science and Automation from Indian Institute of Science Bangalore. He was

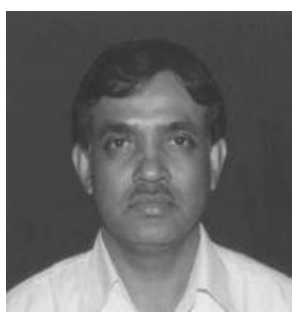
awarded Ph.D. in Economics from Bangalore University and Ph.D. in Computer Science from Indian Institute of Technology, Madras. 